\title{
Reseña del libro: Billetes. Del papel moneda a los billetes de banco en Costa Rica, de Manuel Chacón Hidalgo
}

\author{
Book Review: Billetes. Del papel moneda a \\ los billetes de banco en Costa Rica, by Manuel \\ Chacón Hidalgo
}

\section{Andrea Montero Mora*}

\begin{abstract}
Resumen: Se reseña aquí un trabajo sobre historia económica, centrado en el estudio de la moneda y el dinero. Es un trabajo de divulgación científica que analiza el proceso de consolidación de la moneda en Costa Rica desde el siglo XVIII hasta el año 2018. Esta periodización está ordenada cronológicamente en seis épocas distintas, que concuerdan con los seis capítulos en que se divide el libro. Además de esta periodización de la historia de la moneda, el texto ofrece dos hipótesis sobre los usos de la moneda; en primer lugar como mecanismo de control social, y en segundo lugar como elemento de cambio cultural con la llegada del papel moneda.
\end{abstract}

Palabras claves: reseña bibliográfica; papel moneda; sistemas monetarios; economía; historia; Costa Rica.

\begin{abstract}
A work about economic history is reviewed here, centered on the study of money and coin. It's a work of scientific divulgation that analyses the process of coin consolidation in Costa Rica from the eighteenth century up to the year 2018. This periodization is chronologically ordered in six different eras, that match the six chapters in which the book is divided. In addition to this periodization of the history of coin, the text offers two hypotheses about the usage of coin; firstly, as a mechanism of social control, and secondly as an element of cultural change with the arrival of paper-money.
\end{abstract}

Costarricense. Doctora en Historia Económica por la Universidad de Barcelona (UB), España. Profesora en la Escuela de Historia de la Universidad de Costa Rica (UCR), Costa Rica. Investigadora en el Centro de Investigaciones Históricas de América Central (CIHAC), UCR, Costa Rica. Correo electrónico: andrea.monteromora@ucr.ac.cr. 
Keywords: book reviews; paper money; monetary systems; economy; history; Costa Rica.

$\mathrm{E}$ 1 uso de dinero en efectivo se está limitando gradualmente en casi todos los países — desarrollados y en vías de ello-. El billete o moneda está siendo reemplazado por el dinero digital. Suecia es un caso extremo, y es el país que está más cerca de erradicar el efectivo. Muchos comercios se niegan a recibirlo, y la mayoría de las oficinas bancarias no permiten depositarlo o retirarlo. Las compras por Internet son la norma, el pago con tarjeta es el más frecuente, y cada día son mayores las transacciones por medio de aplicaciones digitales. Actualmente, una tercera parte de los suecos no utilizan billetes ni monedas, e incluso las compras al por menor las realizan con dinero electrónico. ${ }^{1}$ Otros países han seguido el ejemplo sueco lanzando una serie de programas con plazos establecidos para poder realizar sus transacciones sin tener que recurrir a las monedas o los billetes ${ }^{2}$. De acuerdo con el Word Cash Report 2018, Suecia puede considerarse legítimamente, como el país menos dependiendo del efectivo, del mundo, y le siguen en importancia Nueva Zelanda, Corea del Sur, Australia, Canadá, Estados Unidos e Inglaterra. ${ }^{3}$

Son cada vez más frecuentes los artículos académicos, libros y reportes que se publican analizando los alcances y las limitaciones de abandonar el dinero físico y transitar hacia el electrónico. Para los defensores de esta política, el efectivo es el principal causante de la evasión de impuestos y de la economía sumergida e ilegal. Argumentan que con la eliminación del efectivo se favorecerían las políticas monetarias y económicas de los bancos y los gobiernos, se impediría - en el escenario de una crisis o pánico bancario - la retirada masiva de dinero, y se eliminarían los costes de producción, mantenimiento y gestión de la acuñación de monedas y de la emisión de billetes. Para los detractores de esta política, el abandono del dinero tradicional solo favorecería al sector financiero, debido a que los bancos y las empresas de tecnología financiera tendrían el control para supervisar todas las transacciones, porque sería fácil rastrear, lo que podría repercutir en niveles de vigilancia social masiva. Además, esta medida radical iría contra la intimidad de la economía personal y doméstica, provocaría mayor exclusión social y financiera — ciudadanos no bancarizados, sin cuentas, ni tarjetas_-, delimitaría el derecho de propiedad, y afectaría la libertad individual. ${ }^{4}$

1 Nikas Arvidsson, Building a Cashless Society: The Swedish Route to the Future of Cash (Springer International Publishing, 2019).

2 Adrian Athique, «A Great Leap of Faith: The Cashless Agenda in Digital India», New Media \& Society, 21, n. ${ }^{\circ} 8$ (2019): 1697-1713, https://doi.org/10.1177/1461444819831324; Nikola Fabris, «Cashless Society-The Future of Money or a Utopia?», Journal of Central Banking Theory and Practice, 8, n. ${ }^{\circ}$ (2019): 53-66, https://doi.org/10.2478/jcbtp-2019-0003.

3 World Cash Report, https://cashessentials.org/app/uploads/2018/07/2018-world-cash-report.pdf.

4 Los interesados en el tema pueden consultar: Joseph, «Potential negative effects of a cashless society», Journal of Money Laundering Control, 22, n. ${ }^{\circ} 2$ (2019): 350-358, http://dx.doi.org/10.1108/ 
El debate sobre el futuro del dinero en efectivo está abierto y seguirá siendo tema de discusión. Si bien las transferencias electrónicas están ganando terreno, no debemos ignorar que, en la actualidad, la mayor cantidad de operaciones siguen haciéndose con dinero impreso o acuñado. En el marco del actual debate entre dinero físico vs electrónico el libro Billetes. Del papel moneda a los billetes de banco en Costa Rica, del historiador Manuel Chacón Hidalgo ${ }^{5}$ adquiere aún más relevancia y vigencia, porque viene a rescatar la historia de la emisión, circulación y consolidación del papel moneda desde una visión a largo plazo, abordando aspectos económicos, políticos sociales y culturales. Manuel Chacón es doctor en Historia por la Universidad Pablo de Olavide, es curador de Numismática de los Museos del Banco Central, es profesor en la Escuela de Historia de la Universidad de Costa Rica, e investigador en el Centro de Investigaciones Históricas de América Central, del mismo centro universitario. Es un investigador con amplia trayectoria; sus campos de estudio son la historia económica, la historia de la moneda, la historia monetaria, los medios de cambio alternativos, el crédito, la pobreza y la economía de Costa Rica, especialmente para el período colonial y el siglo XIX.

El libro que aquí se está reseñando es el producto de una investigación de muchos años, por parte del autor. La obra se enmarca en el área de la Historia Económica con énfasis en el estudio monetario. Es un texto de divulgación científica, es decir, una publicación de corte informativo, que aborda un tema particular, y está redactado de la manera más sencilla, democrática y comprensible, para asegurar el acceso a la mayor cantidad de públicos interesados en el tema. Si bien tiene un claro carácter divulgativo, es un trabajo que se caracteriza por una investigación científica de base y por una gran solidez argumentativa. En el libro, el objetivo del autor es explicar el proceso de consolidación del uso del papel moneda y de los billetes en Costa Rica, desde el siglo XVIII hasta el 2018. La pregunta general que propone responder es: ¿Cuáles circunstancias históricas posibilitaron el paso a la emisión de billetes por parte de distintos entes privados y estatales hasta llegar a establecerse la Banca Central y la emisión única de billetes por parte del Gobierno costarricense?

La obra la conforman seis capítulos pensados cronológicamente, en los cuales se aborda desde la génesis del papel moneda en el nivel mundial, hasta

JMLC-04-2018-0035; Stan Sater, Financial Privacy in a Cashless Society SSRN, https://ssrn.com/abstract=3367610; Aliya Berkimbayeva,Transition to a Cashless Society: Impact on Economic Activity, https://dspace.cuni.cz/handle/20.500.11956/107724: Kenneth Rogoff, Reduzcamos el papel moneda: una propuesta para disminuir el dinero en efectivo y, con ello, reducir la corrupción, la evasión fiscal, el tráfico de drogas y la economía sumergida (Deusto, España: Ediciones Deusto, 2017); Jonathan McMillan, El fin de la banca: el dinero, el crédito y la revolución digital (Madrid, España: Taurus, 2018); Kenneth Rogoff, The Curse of Cash: How Large-Denomination Bills Aid Crime and Tax Evasion and Constrain Monetary Policy (Princeton University Press, 2017); David Wolman, El fin del dinero, un mundo sin efectivo (México, D.F.: Editorial Océano, 2013).

5 Manuel Chacón Hidalgo, Billetes. Del papel moneda a los billetes de banco en Costa Rica (San José, Costa Rica: Fundación Museos del Banco Central, 2019). 
el origen y la consolidación en Costa Rica con el uso de los billetes bancarios. El autor establece cinco periodos clave en la historia monetaria costarricense: 1) el origen del papel moneda — siglo XVIII a la década de 1850—; 2) los inicios del billete bancario (1858-1896); 3) la reforma monetaria, la ley de bancos y la múltiple emisión (1896-1914); 4) el camino hacia la Banca Central (1914-1950); y 5) la Banca Central y las emisiones de billetes (1950-2018). Utiliza fuentes primarias y secundarias de distintos centros documentales, aporta un material fotográfico de gran valor histórico, y recurre a la descripción/explicación de los hechos que se propuso reconstruir. Si bien en un texto de Historia Económica, Chacón utiliza más el análisis cualitativo que cuantitativo de los datos, lo que responde al carácter divulgativo de la obra y el objeto de ir más allá de un público especializado en temas monetarios.

El primer periodo cubre desde la colonia hasta la república. El autor explica ampliamente, la relación entre el papel moneda y el crédito; pues este último permitió a distintos sectores de la población tener acceso a dinero para desarrollar actividades de inversión y consumo, y palió, de alguna manera, la falta de circulante monetaria. Resalta la diferencia entre los créditos para inversión y los créditos para consumo; aunque en el abordaje faltó detallar en las características de estos créditos - montos, plazos y condiciones-, y ampliar quiénes fueron los que tuvieron acceso - perfiles del prestamista, deudor, fiador-. Cuestiona el tema de la escasez monetaria, presente en la mayoría de los trabajos sobre el periodo colonial, y a manera de hipótesis plantea que la moneda siempre circuló en grupos reducidos, que a su vez procuraron mantener a la mayor parte de la población alejada del acceso a la moneda metálica como un mecanismo de control socioeconómico. Explica el papel de Factoría de Tabacos como un actor clave en la monetización de la economía costarricense, fungiendo casi como banco de depósitos mediante la emisión de libranzas, que funcionaron como una especie de papel moneda. Confirma la existencia de una relación entre escasez monetaria y las emisiones de papel moneda durante el periodo de la independencia y la federación, reconstruyendo los diferentes momentos en los que se tuvo que emitir libranzas y vales, al punto de que se emitieron en mayor cantidad de lo que el gobierno federal pudo amortizar, provocando la pérdida de valor de este papel moneda en el mercado. Finalmente, rescata como dicha escasez fue crónica, y se postergó después de la ruptura de la Federación. De hecho, Costa Rica en varias ocasiones debió recurrir a la emisión de vales con características de papel moneda para sufragar parte de sus gastos, entre ellos, el pago a los funcionarios.

En el segundo periodo Chacón analiza el proceso de consolidación del papel moneda y del billete bancario (1858-1896). Deja claro que los billetes lograron el poder liberatorio muy tarde; entre 1856 y 1896 la aceptación de estos para realizar transacciones era voluntaria. Las monedas de oro y plata tenían 
pleno valor por los metales con que estaban fabricadas, mientras que los billetes eran una especie de certificado que garantizaba que el monto indicado estaba depositado o respaldado por el banco con moneda metálica. Enfatiza que en momentos críticos de mercado - internacional y doméstico-, los billetes fueron una opción para cubrir las necesidades de moneda metálica para las transacciones. Ante la crónica escasez monetaria, las emisiones de billetes resultaban fundamentales para cubrir el faltante de moneda, especialmente, de oro de alta denominación. Confirma que la emisión de papel moneda se instauró casi como costumbre en Costa Rica; entre 1863 y 1884 el Estado costarricense lo emitió, sobre todo para pagar sus obligaciones y financiar proyectos específicos. Y si bien durante la década de 1860, los gobernantes realizaron grandes esfuerzos para enfrentar la deuda interna, esta continuó en aumento, porque se seguía emitiendo para pagar el salario de los empleados públicos. Finalmente, explica las implicaciones de la firma del contrato Soto-Ortuño en el sistema monetario costarricense; a partir de este contrato el Banco de Costa Rica tuvo el monopolio de la emisión hasta la Ley de Bancos de 1900.

En el tercer periodo el autor analiza diferentes temas: la reforma monetaria de 1896, la Ley de Bancos de 1900, y la emisión múltiple (1896-1914). A diferencia de los anteriores, y a pesar de las temáticas tan complejas, el autor le dedica menos páginas a este periodo. Chacón explica la reforma monetaria que acabó con la implantación del patrón oro por parte de Rafael Iglesias, en un claro contexto de la crisis de mercado entre 1896 y 1906. Analiza la Ley de Bancos de 1900, con la que el Banco de Costa Rica pierde el monopolio de la emisión, y a partir de entonces cualquier banco que cumpliera los requisitos impuestos por la ley tenía la posibilidad de emitir; por entonces, los billetes continuaron siendo de uso legal, pero de aceptación voluntaria. Enfatiza en el tema de la emisión múltiple que ocurrió entre 1900 y 1914, periodo en el que circularon billetes de la mayoría de bancos existentes como el de Costa Rica, el Anglo Costarricense, el Comercial y el Mercantil.

En el cuarto periodo, el análisis se enfoca en el proceso de transición del papel moneda hacia su pleno poder liberatorio. Aborda el periodo de entreguerras y explica sus efectos en la economía nacional y el sistema cambiario. Es el momento de las reformas implementadas por Alfredo Gonzáles Flores, entre ellas, la fundación del Banco Internacional. Explica detalladamente, la suspensión de la convertibilidad de los billetes y sus efectos en el sistema monetario, lo que obligó la suspensión del Patrón Oro, advirtiendo que el pleno poder liberatorio por parte de los billetes fue un proceso paulatino, que ocurrió entre 1920 y 1930. Analiza la eliminación por parte del Gobierno de la pluralidad de emisiones y el proceso de centralización en 1922, a través de la Caja de Conversión, que fue creada como una sección del Banco Internacional, pero trabajó de forma independiente. Señala los efectos de la crisis de 1929 en la economía, obligando 
a tener que devaluar el colón con respecto al oro y provocando profundas fisuras en la Caja de Conversión, teniéndose que liquidar este organismo en 1931; fue entonces, cuando el Congreso facultó la emisión al Banco Internacional. Finalmente, aborda la reforma bancaria de 1936 que consistió en implementar una moneda administrada y emitida por el Departamento Emisor, el cual controlaría su oferta adecuándola a las necesidades del país. Así, el Banco Nacional inició la emisión en 1936, primero con fórmulas del Banco Internacional reselladas, y a partir de 1939 con las propias. Fue en 1939 cuando por primera vez se dejaron de incluir en los billetes promesas de pago en moneda acuñada o en moneda extranjera respaldada por oro.

En el quinto y último momento, el autor aborda el nacimiento de la Banca Central y las emisiones de papel moneda a partir de 1950, primero bajo las fórmulas del Banco Nacional, y a partir de 1951 con fórmulas propias. Explica con detalle cómo el Banco Central se convirtió en el organismo rector y promotor del desarrollo de la economía costarricense, de la política monetaria, crediticia y cambiaria, y el único autorizado para emitir monedas y billetes. Profundiza en los cambios de la teoría monetaria en la que se inscribió el Banco Central, donde la emisión monetaria quedaba respaldada por los valores representativos de las fuerzas económicas, partiendo del concepto de que la moneda vale por lo que con ella puede comprarse. Explica la política monetaria del Banco Central en periodos favorables y críticos del mercado, como la bonanza económica entre 1950 y 1970 , y la crisis de los ochenta. Finalmente, enfatiza y aclara que en la actualidad - entiéndase 2018 - la mayor parte de la emisión y la circulación monetaria está compuesta por billetes, ya que el aumento constante en los precios ha provocado que cada día se demande una cantidad mayor de dinero y que cada vez sea más difícil adquirir un bien con monedas.

Podemos resumir este libro en cinco grandes conclusiones: 1) la escasez de moneda obligó a recurrir al uso de papel moneda para la mayoría de las transacciones. Ya a finales del siglo XIX, la circulación del país estuvo compuesta mayoritariamente por billetes, convertibles a la vista en plata; 2) cuando se estableció el Patrón Oro y se decretó la Ley de Bancos, la circulación del papel moneda estaba en un franco proceso de consolidación, y ya existían instituciones bancarias con una amplia trayectoria que contaban con la confianza del público; 3) después de 1914, no se discutió la importancia del papel moneda, más bien se crearon mecanismos para regular y fortalecer su emisión y circulación, aunque sus emisiones debían ser respaldas por dólares convertibles en oro; 4) después de la crisis de 1929, el abandono de los patrones metálicos de respaldo de los billetes y de la acuñación de monedas en metales preciosos puso al papel moneda y a la moneda metálica en igualdad de condiciones para su emisión y circulación, ambos con pleno poder liberatorio y curso legal y forzoso, lo cual generó la consolidación del uso del papel moneda en Costa Rica; y 5) en 1950, se fundó el 
Banco Central de Costa Rica, sobre la base del Departamento Emisor del Banco Nacional y, desde entonces, es el único ente autorizado para emitir moneda.

El autor logra responder la pregunta de partida, y plantea dos grandes hipótesis que pueden servir de base para futuras investigaciones. La primera es con respecto al tema de la escasez monetaria, en este sentido plantea que la moneda siempre circuló en grupos reducidos, que a su vez procuraron mantener a la mayor parte de la población alejada del acceso a la moneda metálica como un mecanismo de control socioeconómico. Sin duda es un planteamiento sugerente, que se tiene que investigar, pues esta dinámica de acaparamiento del circulante monetario es lo que nos permitirá entender la consolidación de las élites en el poder y su permanencia en el tiempo, un tema pendiente en la historiografía costarricense. La segunda es con respecto al componente sociocultural, en relación con la aceptación del uso del billete. Aquí, el autor lanza una serie de preguntas: ¿cómo llegó a consolidarse el uso de papel moneda, si la sociedad estaba acostumbrada a usar monedas metálicas cuyo valor nominal, facial, por lo general era igual al valor intrínseco?, ¿por qué un individuo estaba dispuesto a aceptar papel moneda de un ente privado, como los bancos, que se fundaron en la segunda mitad del siglo XIX? ¿cómo fue que el público siguió teniendo confianza para depositar dinero, realizar transacciones y recibir papel moneda a pesar de la quiebra de algunos bancos? De forma sucinta, Chacón las responde señalando tres aspectos: el respaldo que daba el Estado a estas emisiones, la confianza de los usuarios clientes hacia el «personaje banquero»; la casi imposición. Sin duda el proceso tuvo que ser más complejo, debieron existir controversias entre diferentes autores económicos, políticos e institucionales que se tendrán que estudiar, pero es un planteamiento provocador para futuras investigaciones.

La lectura de la obra despierta la curiosidad y tiene la virtud de suscitar interrogantes. A futuro es importante que se aborde desde la perspectiva histórica: 1) las consecuencias económicas y sociales de la emisión desenfrenada de papel moneda en un contexto de crisis política y económica durante la Federación Centroamericana; 2) la dinámica de acaparamiento del circulante monetario por parte de ciertos grupos, para entender el proceso de conformación de las élites económicas y su permanencia en el tiempo - perfiles del prestamista, deudor, fiador-; 3) aclarar cuánto de la emisión se destinó a proyectos prioritarios y cuánto al pago de los salarios del sector público, 4) profundizar en el ambiente de inestabilidad que causó la suspensión de la convertibilidad, y el efecto de la suspensión en la población que había confiado sus ahorros a los bancos; 5) y estudiar el componente sociocultural que condujo a la aceptación del uso del papel moneda.

Concluyo diciendo que es un libro de síntesis que termina respondiendo la pregunta y el objetivo de partida. Para lograrlo hubo un arduo trabajo de investigación científica, que incluyó el planteamiento de hipótesis, la búsqueda de 
fuentes y la confrontación de datos. En la obra, se cuidó con gran detalle el aspecto de edición, estilo y forma, lo que permite una lectura fluida. A pesar de trabajar temas complejos, el autor tuvo la habilidad de presentarlos con un lenguaje sencillo, de modo que la obra es de fácil comprensión para un público variado que pueda estar interesado en temas de historia económica, monetaria o numismática. El trabajo es un buen ejemplo de cómo la investigación científica realizada en la academia se puede no solo difundir - libros y revistas académicos-, sino también divulgar a distintos públicos meta.

\section{Bibliografía}

Arvidsson, Nikas. Building a Cashless Society: The Swedish Route to the Future of Cash. Springer International Publishing, 2019.

Athique, Adrian. «A Great Leap of Faith: The Cashless Agenda in Digital India». New Media \& Society, 21, n. ${ }^{\circ} 8$ (2019): 1697-1713. https://doi. org/10.1177/1461444819831324.

Berkimbayeva, Aliya. Transition to a Cashless Society: Impact on Economic Activity. https://dspace.cuni.cz/handle/20.500.11956/107724.

Chacón Hidalgo, Manuel. Billetes. Del papel moneda a los billetes de banco en Costa Rica. San José, Costa Rica: Fundación Museos del Banco Central, 2019.

Fabris, Nikola. «Cashless Society-The Future of Money or a Utopia?». Journal of Central Banking Theory and Practice, 8, n. ${ }^{\circ} 1$ (2019): 53-66. https:// doi.org/10.2478/jcbtp-2019-0003.

McMillan, Jonathan. El fin de la banca: el dinero, el crédito y la revolución digital. Madrid, España: Taurus, 2018.

Rogoff, Kenneth. Reduzcamos el papel moneda: una propuesta para disminuir el dinero en efectivo y, con ello, reducir la corrupción, la evasión fiscal, el tráfico de drogas y la economía sumergida. Deusto, España: Ediciones Deusto, 2017.

Rogoff, Kenneth. The Curse of Cash: How Large-Denomination Bills Aid Crime and Tax Evasion and Constrain Monetary Policy. Princeton University Press, 2017.

Sater, Stan. Financial Privacy in a Cashless Society SSRN. https://ssrn.com/ abstract $=3367610$.

Wilfrido Rivera, Joseph. «Potential negative effects of a cashless society». Journal of Money Laundering Control, 22, n. ${ }^{\circ} 2$ (2019): 350-358. http://dx.doi. org/10.1108/JMLC-04-2018-0035.

Wolman, David. El fin del dinero, un mundo sin efectivo. México, D.F.: Editorial Océano, 2013. 